\title{
Sclerosing encapsulating peritonitis in a long-term propranolol user
}

\author{
Se Hui Noh ${ }^{1 *}$, Byong Duk Ye ${ }^{1,2}$, Hoonsub So ${ }^{1}$, Yu Seok Kim ${ }^{1 *}$, Dong Jin Suh ${ }^{1 *}$, Sang Nam Yoon ${ }^{3 *}$ \\ ${ }^{1}$ Department of Gastroenterology, ${ }^{2}$ Inflammatory Bowel Disease Center, ${ }^{3}$ Department of Colon \& Rectal Surgery, Asan Medical Center, \\ University of Ulsan College of Medicine, Seoul, Korea
}

Sclerosing encapsulating peritonitis (SEP) is a rare cause of bowel obstruction that is characterized by the encasement of the small bowel by a thick, whitish, and fibrous membrane. The pathophysiology of SEP is poorly understood and preoperative diagnosis is difficult. Previous reports suggest that SEP may be linked to the chronic use of $\beta$-adrenergic blockers. A 46-yearold man with liver cirrhosis and end-stage renal disease on hemodialysis presented with recurrent abdominal pain and borborygmi. He had been taking propranolol to prevent bleeding from gastroesophageal varices for the past 15 years. Abdominal computed tomography showed ileal loops encapsulated by soft tissue with dilatation of the proximal small bowel on the right side of the abdomen. Barium follow-through showed conglomerated distal ileal loops with a cauliflower-like appearance. Explorative laparotomy revealed a thick, fibrous, whitish capsule encapsulating the ileal loops. The covering membrane was dissected and excised, resulting in an improvement in symptoms after surgery. Accordingly, a final diagnosis of SEP was made. Due to the lack of other apparent causes for SEP, we conclude that in this case, the long-term use of propranolol may be associated with the development of SEP. (Intest Res 2016;14:375-378)

Key Words: Abdominal pain; Peritonitis; Propranolol

\section{INTRODUCTION}

Sclerosing encapsulating peritonitis (SEP) is a rare cause of intestinal obstruction that is characterized by the partial or complete encasement of the small bowel by a thick, white, and fibrous membrane. SEP was first described as "peritonitis chronica fibrosa encapsulate" in 1907. ' SEP can be classified as either idiopathic or secondary, though the pathogenesis of

Received April 17, 2015. Revised August 8, 2015.

Accepted August 24, 2015.

Correspondence to Byong Duk Ye, Department of Gastroenterology and Inflammatory Bowel Disease Center, Asan Medical Center, University of Ulsan College of Medicine, 88 Olympic-ro 43-gil, Songpa-gu, Seoul 05505, Korea. Tel: +82-2-3010-3181, Fax: +82-2-476-0824, E-mail: bdye@amc. seoul.kr

${ }^{*}$ Current affiliation of Se Hui Noh and Dong Jin Suh is the Vievis NAMUH Hospital, Seoul, Korea, current affiliation of Yu Seok Kim is KMG Hospital for Internal Diseases, and current affiliation of Sang Nam Yoon is Department of Surgery, Hallym University Kangnam Sacred Heart Hospital, Hallym University College of Medicine, Seoul, Korea.

Financial support: None. Conflict of interest: None. both forms is unclear. Idiopathic SEP has been described in adolescent girls, leading to hypothesis that retrograde menstruation or retrograde infection through the fallopian tubes may be the underlying mechanism of SEP., ${ }^{2,3}$ Secondary SEP occurs most frequently in patients on continuous ambulatory peritoneal dialysis (CAPD)., ${ }^{4,5}$ In addition to CAPD, chronic inflammatory conditions, orthotopic liver transplantation, and $\beta$-adrenergic blocker use have been suggested to be predisposing factors for SEP. ${ }^{6}$ Here, we describe a patient with liver cirrhosis (LC) who developed typical features of SEP after the long-term use of propranolol.

\section{CASE REPORT}

A 46-year-old man presented with cramping abdominal pain and borborygmi, which aggravated with food and water intake. Over the previous 12 months, he had lost $6 \mathrm{~kg}$ of body weight. He reported two similar episodes of abdominal pain during the previous 14 months. The previous two episodes

\footnotetext{
(c) Copyright 2016. Korean Association for the Study of Intestinal Diseases. All rights reserved.

This is an Open Access article distributed under the terms of the Creative Commons Attribution Non-Commercial License (http://creativecommons.org/licenses/by-nc/4.0)

which permits unrestricted non-commercial use, distribution, and reproduction in any medium, provided the original work is properly cited.
} 
were conservatively managed with Levin tube drainage with nil per os, and the symptoms improved thereafter. He was diagnosed with LC associated with chronic hepatitis B 18 years ago. Additionally, 15 years ago, he started taking oral propranolol (40 mg/day) for the prophylaxis of gastroesophageal variceal bleeding. Further, 14 months earlier, he was diagnosed with end-stage renal disease due to uncontrolled hypertension and started to undergo hemodialysis. He had no history of spontaneous bacterial peritonitis (SBP), abdominal surgery, or CAPD. At the time of presentation, the patient was taking propranolol, nifedipine, valsartan, pantoprazole, oral iron, calcium carbonate, and multivitamins.

On physical examination, the patient was afebrile and hemodynamically stable. The abdomen was distended with in-

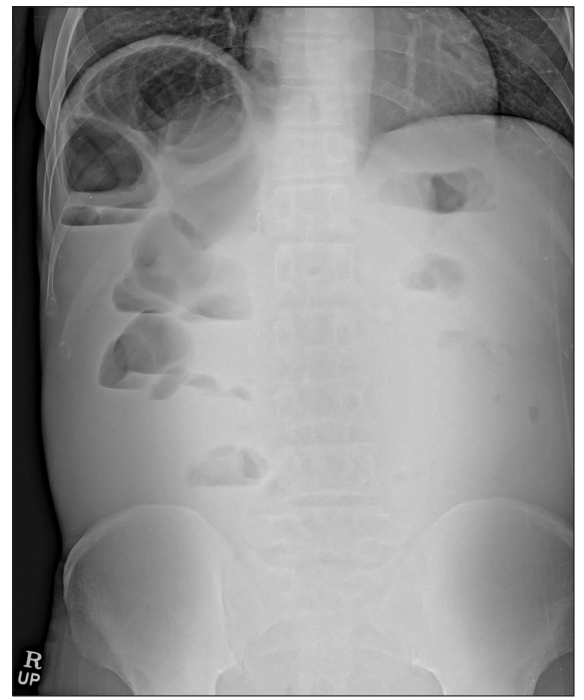

Fig. 1. Plain abdominal radiography. Small bowel dilatation and airfluid levels are seen in the right side of the patient's abdomen (upright position). creased bowel sounds. Percussion of the abdomen resulted in audible tympanic sounds. Shifting dullness was present and splenomegaly was detected. Laboratory findings were as follows: white blood cell count, $5,100 / \mathrm{mm}^{3}$; hemoglobin, 9.6 $\mathrm{g} / \mathrm{dL}$; platelet count, $107 \times 10^{3} / \mathrm{mm}^{3}$; blood urea nitrogen, 54 $\mathrm{mg} / \mathrm{dL}$; creatinine, $7.9 \mathrm{mg} / \mathrm{dL}$; albumin, $2.6 \mathrm{~g} / \mathrm{dL}$; aspartate transaminase, $21 \mathrm{IU} / \mathrm{L}$; alanine transaminase, $10 \mathrm{IU} / \mathrm{L}$; alkaline phosphatase, $45 \mathrm{IU} / \mathrm{L}$; CRP, $3.67 \mathrm{mg} / \mathrm{dL}$; and prothrombin time, $64.3 \%$ (1.27 INR). Diagnostic paracentesis showed no evidence of SBP. Plain abdominal radiography showed small bowel dilatation with air-fluid levels on the right side of the abdomen (Fig. 1). Abdominal CT showed large amounts of ascites and ileal loops encapsulated by soft tissue with dilatation of the proximal small bowel on the right side of the abdomen (Fig. 2). Barium follow-through showed conglomerated distal ileal loops with a cauliflower-like appearance. Palpation did not separate these conglomerated bowel loops, but allowed passage of contrast medium (Fig. 3). On the basis of these findings, the patient was diagnosed with partial small bowel obstruction due to SEP. As his symptoms were recurrent and did not improve with conservative management, exploratory laparotomy was performed, which showed a thick, fibrous, and white capsule encapsulating the ileal loop (Fig. 4A). The capsule was dissected and excised, releasing a 1.5-m section of the small bowel (Fig. 4B). Histopathological examination of the capsule revealed a dense fibrous wall without epithelial lining (Fig. 5). The postoperative period was uneventful, and symptoms improved after the procedure. Propranolol was stopped after surgery. The patient has been receiving conservative management for LC and hemodialysis for 58 months after the surgery, without recurrence of bowel obstruction.
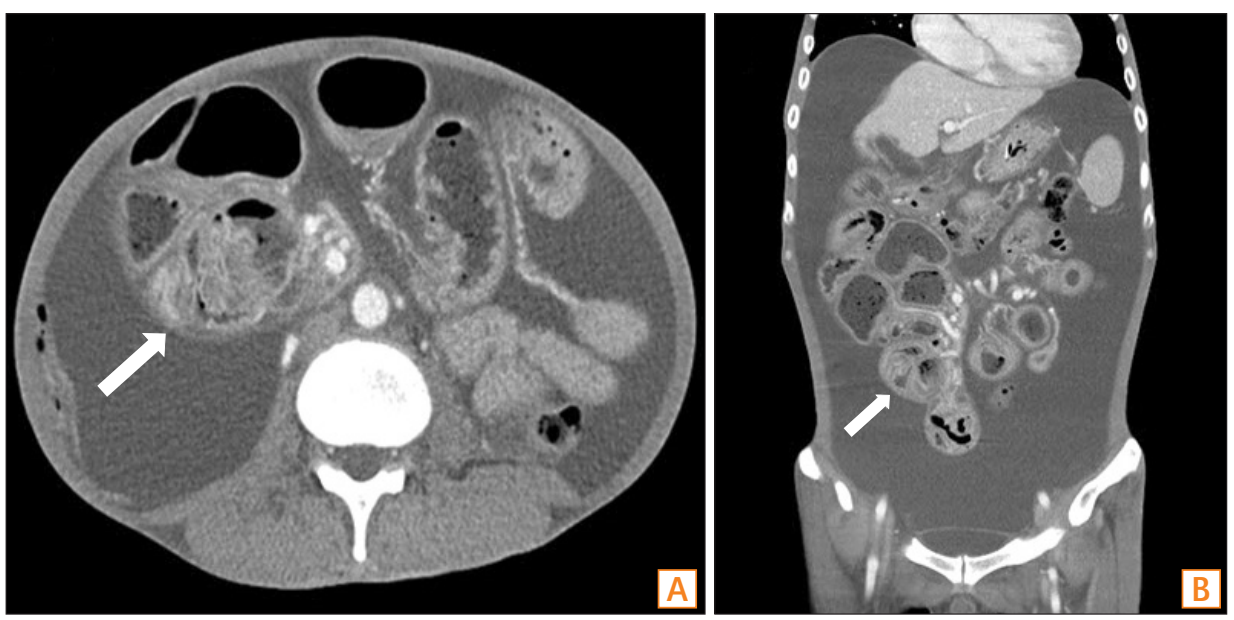

Fig. 2. Abdominal CT findings. Abdominal CT showing large amounts of ascites and ileal loops encapsulated by soft tissue (arrows) with dilatation of the upstream small bowel on the right side of the abdomen. (A) Transverse section. (B) Coronal section. 


\section{DISCUSSION}

SEP is a rare clinical entity that can cause acute, subacute, or chronic small intestinal obstruction. This condition is characterized by a thick, fibrotic, and cocoon-like mem-

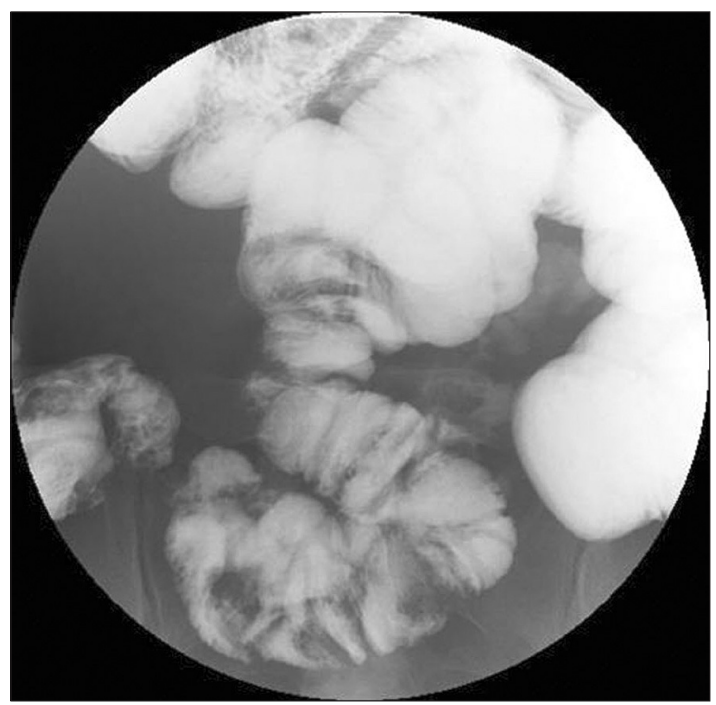

Fig. 3. Barium follow-through (BFT) finding. BFT showing conglomerated distal ileal loops with a cauliflower-like appearance. brane, which partially or totally encases the small bowel. ${ }^{2,6}$ Long-term peritoneal stimulation, which can occur with CAPD, is thought to be a primary cause for secondary SEP., ${ }^{4,5}$ Other reported associations include abdominal tuberculosis, ${ }^{7}$ long-term use of $\beta$-adrenergic blockers, ${ }^{6,8-13}$ ventriculoperitoneal or peritoneovenous shunts in patients with $\mathrm{LC}^{14}$ orthotropic liver transplantation, ${ }^{15}$ and sarcoidosis. ${ }^{16}$

The association between SEP and the $\beta$-adrenergic blocker, practolol, was first reported in $1974 .^{8}$ Other $\beta$-adrenergic blockers associated with SEP include timolol, ${ }^{9}$ metoprolol, ${ }^{12}$ and atenolol. ${ }^{13}$ There have been two reports on SEP associated with propranolol administered for the treatment of chronic angina pectoris, ${ }^{10,11}$ and one on SEP in a patient with LC who was taking a propranolol for over 4 years. ${ }^{6}$ Two mechanisms by which $\beta$-adrenergic blockers can cause SEP have been suggested, including enhanced collagen production, or an allergic reaction to the drug. ${ }^{11,17}$ Our patient had been taking propranolol for 15 years and had no history of SBP or CAPD. Moreover, there was no evidence of other reported conditions commonly linked to SEP. Thus, we suspect that propranolol may have played a role in the development of secondary SEP in our patient.

Preoperative diagnosis of SEP is challenging, and a consid-
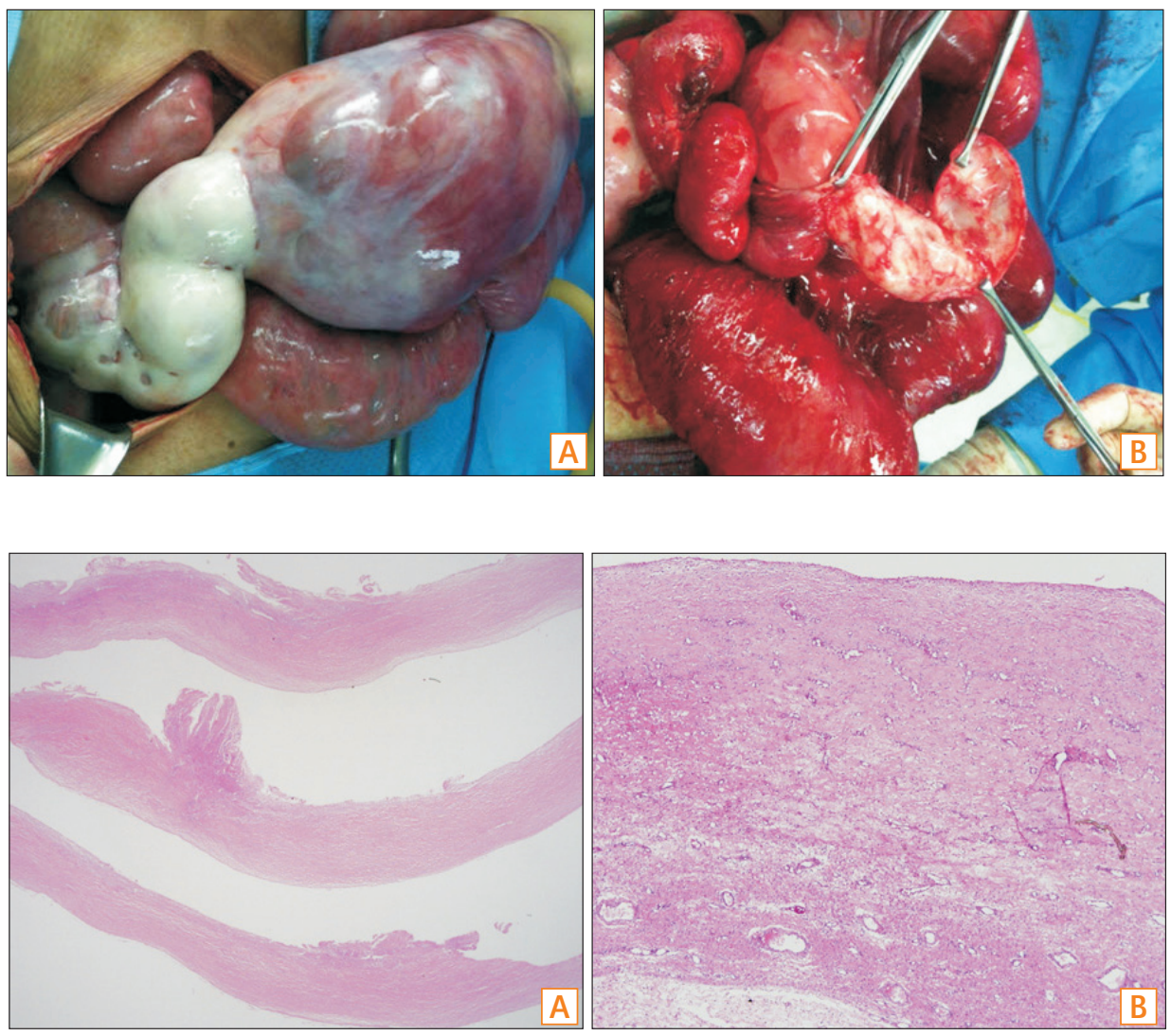

Fig. 4. Surgical findings. (A) Explorative laparotomy showing a thick, fibrous, and white capsule encapsulating ileal loops. (B) Dissection and excision of the capsule released a $1.5 \mathrm{~m}$-long loops of ileum from the capsule.

Fig. 5. Microscopic findings of the resected capsule. A dense fibrous wall without epithelial lining is seen $(A, H \& E, \times 40 ; B, H \& E$, $\times 100$ ). 
erable number of patients are diagnosed with SEP at laparotomy. Accordingly, clinicians should evaluate the possibility of SEP in cases with compatible history and symptoms of this condition to ensure the appropriate management of patients. Radiologically, barium follow-through studies usually show the ileal loops clumped together within a sac having a cauliflower-like appearance. ${ }^{3}$ Abdominal CT can reveal small bowel loops congregated to the center of the abdomen and encased by a soft tissue density mantle, making it look like a cocoon. ${ }^{18}$ Consistent with these observations, typical features of conglomerated and encapsulated bowel loops on both barium study and CT, were seen in the present case.

SEP can be managed medically or surgically. Surgical intervention usually includes the careful and complete removal of the covering membrane and adhesiolysis of the dense interbowel adhesions. ${ }^{6}$ According to one report, SEP can be successfully treated by total enterolysis and the oral administration of prednisolone $5 \mathrm{mg} /$ day. ${ }^{19}$ Others reported a lower mortality rate after treatment with tamoxifen in patients with PD-related SEP. ${ }^{20}$ Conservative therapy, including long-term intravenous hyperalimentation and Levin tube drainage, may be an alternative to surgery in patients with sub-acute intestinal obstruction. ${ }^{6}$ Our patient was initially managed conservatively, with nil per os and parenteral nutrition, because of the risk associated with surgery related to poor hepatic function. Surgical intervention was later required, however, because his symptoms did not improve with conservative management. Prednisolone was not given to our patient due to a history of chronic hepatitis B.

In conclusion, SEP is a rare cause of intestinal obstruction with an uncertain pathophysiology. SEP should be considered in the differential diagnosis of patients with small bowel obstruction who receive long-term treatment with $\beta$-adrenergic blockers.

\section{REFERENCES}

1. Owtschinnikow P. Peritonitis chronica fibrosa incapsulata. Arch Klin Chir 1907;83:623-634.

2. Foo KT, Ng KC, Rauff A, Foong WC, Sinniah R. Unusual small intestinal obstruction in adolescent girls: the abdominal cocoon. Br J Surg 1978;65:427-430.

3. Sieck JO, Cowgill R, Larkworthy W. Peritoneal encapsulation and abdominal cocoon: case reports and a review of the literature. Gastroenterology 1983;84:1597-1601.

4. Bradley JA, McWhinnie DL, Hamilton DN, et al. Sclerosing obstructive peritonitis after continuous ambulatory peritoneal dialysis. Lancet 1983;2:113-114.
5. Holland P. Sclerosing encapsulating peritonitis in chronic ambulatory peritoneal dialysis. Clin Radiol 1990;41:19-23.

6. Kalra S, Atia A, McKinney J, Borthwick TR, Smalligan RD. Sclerosing encapsulating peritonitis associated with propranolol usage: a case report and review of the literature. J Dig Dis 2009;10:332-335.

7. Kaushik R, Punia RP, Mohan H, Attri AK. Tuberculous abdominal cocoon: a report of 6 cases and review of the Literature. World J Emerg Surg 2006;1:18.

8. Brown P, Baddeley H, Read AE, Davies JD, McGarry J. Sclerosing peritonitis, an unusual reaction to a beta-adrenergic-blocking drug (practolol). Lancet 1974;2:1477-1481.

9. Baxter-Smith DC, Monypenny IJ, Dorricott NJ. Sclerosing peritonitis in patient on timolol. Lancet 1978;2:149.

10. Harty RF. Sclerosing peritonitis and propranolol. Arch Intern Med 1978;138:1424-1426.

11. Ahmad S. Sclerosing peritonitis and propranolol. Chest 1981;79:361-362.

12. Clark CV, Terris R. Sclerosing peritonitis associated with metoprolol. Lancet 1983;1:937.

13. Grefberg N, Nilsson P, Andreen T, et al. Sclerosing obstructive peritonitis, beta-blockers, and continuous ambulatory peritoneal dialysis. Lancet 1983;322:733-734.

14. Stanley MM, Reyes CV, Greenlee HB, Nemchausky B, Reinhardt GF. Peritoneal fibrosis in cirrhotics treated with peritoneovenous shunting for ascites: an autopsy study with clinical correlations. Dig Dis Sci 1996;41:571-577.

15. Maguire D, Srinivasan P, O'Grady J, Rela M, Heaton ND. Sclerosing encapsulating peritonitis after orthotopic liver transplantation. Am J Surg 2001;182:151-154.

16. Ngô Y, Messing B, Marteau P, et al. Peritoneal sarcoidosis: an unrecognized cause of sclerosing peritonitis. Dig Dis Sci 1992;37:1776-1780.

17. Berg RA, Moss J, Baum BJ, Crystal RG. Regulation of collagen production by the beta-adrenergic system. J Clin Invest 1981;67:1457-1462.

18. Hur J, Kim KW, Park MS, Yu JS. Abdominal cocoon: preoperative diagnostic clues from radiologic imaging with pathologic correlation. AJR Am J Roentgenol 2004;182:639-641.

19. Yamamoto S, Sato Y, Takeishi T, Kobayashi T, Hatakeyama K. Sclerosing encapsulating peritonitis in two patients with liver cirrhosis. J Gastroenterol 2004;39:172-175.

20. Korte MR, Fieren MW, Sampimon DE, et al. Tamoxifen is associated with lower mortality of encapsulating peritoneal sclerosis: results of the Dutch Multicentre EPS Study. Nephrol Dial Transplant 2011;26:691-697. 